\title{
Elemental Image Synthesis for Integral Imaging Using Phase-shifting Digital Holography
}

\author{
Min-Ok Jeong, Nam Kim, and Jae-Hyeung Park* \\ School of Electrical \& Computer Engineering, Chungbuk National University \\ 410 SungBong-Ro, Heungduk-Gu, Cheongju-Si, Chungbuk, 361-763, Korea
}

(Received October 8, 2008 : revised December 3, 2008 : accepted December 3, 2008)

\begin{abstract}
We propose a method generating elemental images for the integral imaging using 4-step phaseshifting digital holography. Phase shifting digital holography is a way recording the digital hologram by changing the phase of the reference beam and extracting the complex field of the object beam. Since all 3D information is captured by phase-shifting digital holography, the elemental images for any specifications of the lens array can be generated from single phase-shifting digital holography. In experiment, phase-shifting is achieved by rotating half- and quarter- wave plates and the resultant interference patterns are captured by a $3272 \times 2469$ pixel CCD camera with 27 $\mu \mathrm{m} \times 27 \mu \mathrm{m}$ pixel size.
\end{abstract}

Keywords: Digital holography, Phase shifting holography

OCIS codes : (090.0090) Holography; (100.0100) Image processing; (090.2880) Holographic interferometry; (130.0130) Integrated optics

\section{INTRODUCTION}

Imaging of three-dimensional(3D) objects is important for various applications including display. Holography is one of the useful 3D imaging methods since it can record full $3 \mathrm{D}$ information of the object. Photographic recording and optical reconstruction requiring chemical processing, however, have been problematic for conventional holography [1]. Overcoming the disadvantages of the conventional holography, digital holography has been proposed recently. The digital holography uses Charge Coupled Devices(CCD) for recording holographic patterns and reconstructs 3D object images by numerical Fresnel propagation. The whole process is simple because of the use of the CCD instead of the holographic film, the processing time is within the practical range due to the development of the computing power. With these advantages, the digital holography has been used in various practical applications including spatial filtering, inspection of optical systems, tests of laser beams and reconstruction of $3 \mathrm{D}$ images, etc [2-5].

Integral imaging is one of the attractive autostereoscopic 3D display techniques. Using a lens array,

*Corresponding author: jh.park@cbnu.ac.kr integral imaging can display full parallax, auto-stereoscopic $3 \mathrm{D}$ images continuously within a viewing angle without any eye fatigue [6-9]. One problem of integral imaging is that the lens array should be the same for the pickup and the display process. If the lens array of different specifications is used for the pickup and display process, the 3D image will be distorted severely. Generally, it is hard to expect that all the integral imaging display systems have lens arrays of the same specifications since it should vary according to the display environment and desired applications. Many variations of the integral imaging display for enhancing the viewing angle, available depth range and the viewing resolution also require special lens arrays or elemental images matched to their own purpose [10-14]. It is obviously not practical to repeat capturing the elemental images for the different types of display systems. Therefore it is necessary to devise a method to capture the 3D information of the object by a single pickup process and process the captured $3 \mathrm{D}$ information to synthesize the specific elemental images for various different display systems. Although a scaling technique of the elemental images has been reported to deal with this problem, it does not provide full freedom in the lens array specification [7-9]. 
Hologram is a good alternative for direct capturing. Since a hologram captures full 3D information about the object, it is possible to synthesize the desired elemental images from the single hologram of the object. In this report, we propose a method generating elemental images for integral imaging using phase-shifting digital holography. The proposed method obtains the complex field of the object wave at the CCD plane using 4-step phase shifting digital holography. By propagating the obtained complex field numerically assuming a virtual lens array, the proposed method synthesizes the elemental images. Since we can set the specifications and the location of the lens array arbitrarily in the numerical imaging, we can generate the elemental images corresponding to any integral imaging display systems from single capture of phase shifting digital holography.

The proposed method is closely related to Javidi et al.'s method [15]. Javidi et al. also synthesizes the elemental images from the object complex field obtained by the 4 -step phase shifting digital holography. Their method, however, generates the elemental image by projecting and accumulating the object plane images reconstructed at various depths which sweep the object space onto the elemental image plane assuming the pinhole array based on ray optics. Therefore it is not possible to synthesize the elemental images for the lens array of the arbitrary specifications including the aperture size of the elemental lens, and irregular curvature of the elemental lens surface. The proposed method can deal with the lens array of any specifications since it is fully based on wave optics and does not use a pinhole approximation. Kim et al [16]. also proposed an elemental image generation method from the hologram. The elemental image plane, however, is fixed in their method and the elemental image synthesis for arbitrary lens array locations and specifications, which is easily realized in the proposed method, is not presented.

In the following, we explain the principle of the proposed method and provide the experimental results for verification.

\section{PRINCIPLE}

The first step of the proposed method is the capture of the object complex field using phase-shifting digital holography. Figure 1 is the coordinate system for the numerical reconstruction of phase-shifting digital holography. The object wave at the CCD camera plane $U(x, y)$ is represented as a Fresnel Transform of the complex field at the object plane $U_{o}\left(x^{\prime}, y^{\prime}\right)$ by

$$
\begin{aligned}
U(x, y) & =\iint U_{o}\left(x^{\prime}, y^{\prime}\right) \exp \left[i k \frac{\left(x-x^{\prime}\right)^{2}+\left(y-y^{\prime}\right)^{2}}{2 z_{o}}\right] d x^{\prime} d y^{\prime} \\
& =A(x, y) \exp [i \phi(x, y)]
\end{aligned}
$$

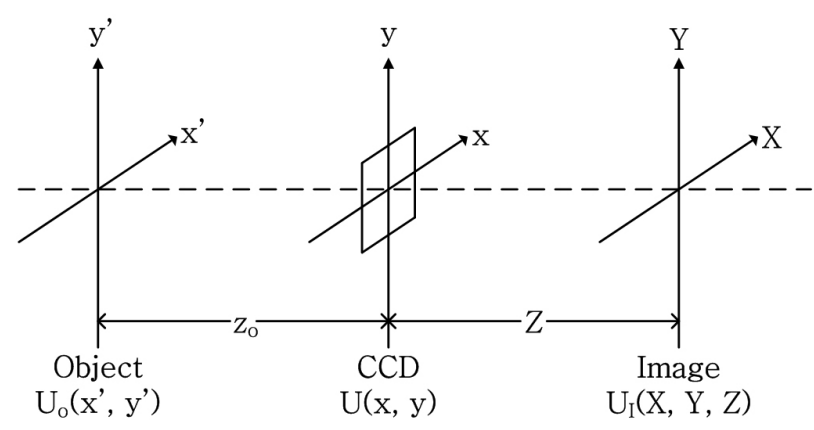

FIG. 1. The coordinate system for the numerical reconstruction.

where $\lambda$ is the wave length, $k$ is the wave number given by $\mathrm{k}=2 \pi / \lambda$ and $z_{o}$ is the distance from the object plane to the CCD camera. A plane reference wave whose complex amplitude is represented by $U_{r}(x, y ; \alpha)=A_{r}(x, y)$ $\exp \left[i \phi_{r}(x, y)\right] \exp [i a]$ is superimposed upon the object wave. Since the interference patterns detected by CCD camera have only the intensity value, the resultant interference intensity recorded by the CCD camera is given by

$$
\begin{aligned}
& I(x, y ; \alpha)=\left|U(x, y)+U_{r}(x, y)\right|^{2} \\
& \quad=[A(x, y)]^{2}+\left[A_{r}(x, y)\right]^{2}+2 A(x, y) A_{r}(x, y) \cos \left[\phi(x, y)-\phi_{r}(x, y)-\alpha\right]
\end{aligned}
$$

where $a$ is the mean phase difference between the object and the reference waves. By using the phaseshifting procedure in which the reference phase is shifted by a step of $\pi / 2$, we can derive the object phase $\left(\phi_{E}\right)$ such that

$$
\phi_{E}(x, y)=\phi(x, y)-\phi_{r}(x, y)=\tan ^{-1}\left[\frac{I(x, y ; \pi / 2)-I(x, y ; 3 \pi / 2)}{I(x, y ; 0)-I(x, y ; \pi)}\right]
$$

The object amplitude, $A_{E}(x, y)$, can be calculated from the following equation,

$$
A_{E}(x, y)=A(x, y) A_{r}(x, y)=\frac{1}{4} \frac{I(x, y ; 0)-I(x, y ; \pi)}{\cos \left[\phi(x, y)-\phi_{r}(x, y)\right]}
$$

where the argument of the cosine function in the denominator is directly obtained from Eq. (3) [17].

If we use a plane reference wave, the amplitude $A_{r}(x, y)$ and the phase $\phi_{r}(x, y)$ of the reference wave can be considered as constants. Hence, by using Eqs. (3) and (4), we can derive the complex amplitude of the object wave $(U)$ at the CCD plane [18]. Since the complex object field is obtained at the CCD plane, we can calculate the object field at any plane by numerically propagating the obtained object field. The 


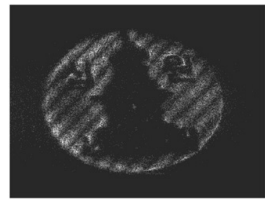

(a)

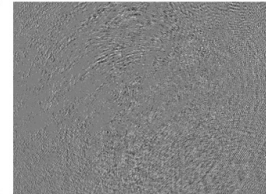

(b)

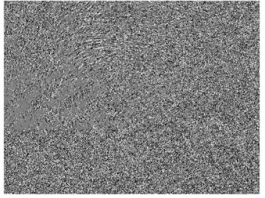

(c)
FIG. 2. The reconstruction of hologram: (a) Reconstructed image, (b) Amplitude and (c) Phase.

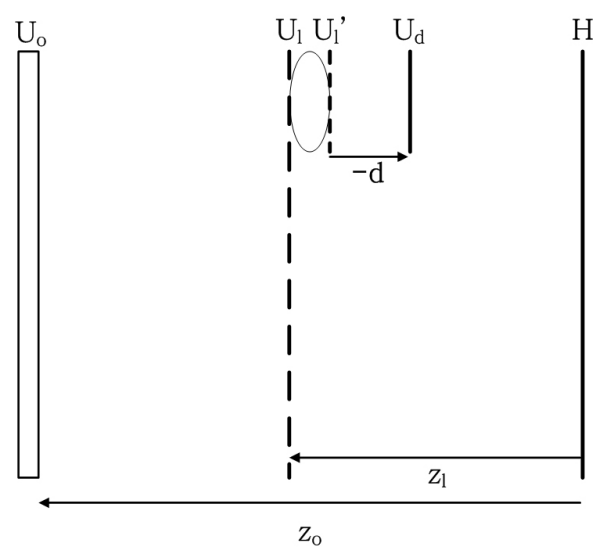

FIG. 3. Schematic geometry of the elemental image reconstruction

numerical propagation of the captured object field $U(x, y)$, the reconstruction field $\left(U_{I}\right)$, is calculated by

$$
\begin{aligned}
U_{I}(X, Y, Z) & =\iint U(x, y) \exp \left[i k \frac{(X-x)^{2}+(Y-y)^{2}}{2 Z}\right] d x d y \\
& =\iint A_{E}(x, y) \exp \left[i \phi_{E}(x, y)\right] \exp \left[i k \frac{(X-x)^{2}+(Y-y)^{2}}{2 Z}\right] d x d y \\
& =\iint A(x, y) \exp [i \phi(x, y)] \exp \left[i k \frac{(X-x)^{2}+(Y-y)^{2}}{2 Z}\right] d x d y
\end{aligned}
$$

where $A_{E}(x, y)=A(x, y) A_{r}(x, y)=A(x, y) \quad$ and $\quad \phi_{E}(x, y)=\phi$ $(\mathrm{x}, \mathrm{y})-\phi_{r}(x, y)=\phi(x, y)$ are used since the amplitude $A_{r}(x, y)$ and the phase $\phi_{r}(x, y)$ of the plane reference wave can be considered as constants. For one example of the numerical propagation, if we set $Z=-z_{0}$, then we will get the original object field $U_{o}(x, y)$ at the object plane, i.e.

$$
U_{I}\left(X, Y,-z_{o}\right)=U_{o}(X, Y)
$$

Figure 2 shows one example of the complex object field at the CCD plane, which is captured using the phase-shifting digital holography, and the original object field at the object plane which is obtained by numerical propagation.

The second step of the proposed method is the synthesis of the elemental images for integral imaging using the complex object field $U(x, y)$ captured at the CCD plane. Figure 3 is the schematic geometry for the elemental image synthesis. Figure 3 depicts only one lens for simplicity, but its extension to the lens array is straightforward. In order to obtain the elemental image which is denoted by $U_{d}$ in Fig. 3, we first calculate the optical field $U_{l}$ at the front side of the virtual lens by numerically propagating $U$ by a distance $z_{l}$ using Eq. (5). The optical field $U_{l}^{\prime}$ right behind the lens is given by

$$
U_{l}^{\prime}(X, Y)=U_{l}\left(X, Y, \mathbf{Z}_{1}\right) P(X, Y) \exp \left[-i k \frac{\left(X-x_{o}\right)^{2}+\left(Y-y_{o}\right)^{2}}{2 f}\right]
$$

where, $P(X, Y),\left(x_{0}, y_{0}\right)$, and $f$ are the pupil function, the transverse location and the focal length of the virtual lens, respectively. The elemental image is finally obtained by numerically propagating $U_{l}^{\prime}$ over distance $-d[19]$.

$$
U_{d}(X, Y,-d)=\iint U_{l}{ }^{\prime}(X, Y) \exp \left[-i k \frac{(X-x)^{2}+(Y-y)^{2}}{2 d}\right] d x d y
$$

Note that the sign of the exponential is different from Eq. (5) because of the opposite direction against $Z$. At this time, $d$ can be determined by the lens' law, such as

$$
\begin{aligned}
& \frac{1}{f}=\frac{1}{z_{o}-z_{l}}+\frac{1}{d} \\
& d=\frac{\left(z_{o}-z_{l}\right) f}{\left(z_{o}-z_{l}\right)-f}
\end{aligned}
$$

where $z_{0}$ is the distance between the plane of CCD camera and the object and $z_{l}$ is the distance between the $U_{l}$ plane and the $U$ plane in Fig. 3. By repeating this process for all elemental lenses, the whole elemental image can be obtained.

The proposed method can generate the elemental images for any lens array specifications including the focal length, elemental lens size, number of the elemental lenses and elemental lens arrangement. The resolution and the viewing angle of the elemental image, however, are limited by the sampling interval and the viewing angle of the complex object field captured at the CCD plane. The viewing angles of the object field captured at the CCD camera plane, $\theta_{x}$ and $\theta_{y}$ are given by

$$
\begin{aligned}
& \theta_{x}=2 \tan ^{-1} \frac{\delta_{x} N_{x} / 2}{z_{o}} \\
& \theta_{y}=2 \tan ^{-1} \frac{\delta_{y} N_{y} / 2}{z_{o}}
\end{aligned}
$$

where pixel size of the CCD camera are $\delta_{x} \times \delta_{y}$ and the number of pixels are $N_{x} \times N_{y}$.

The sampling interval varies at each plane. The sampling interval of $U_{l}$ plane, $\Delta x$ and $\Delta y$, are given by 


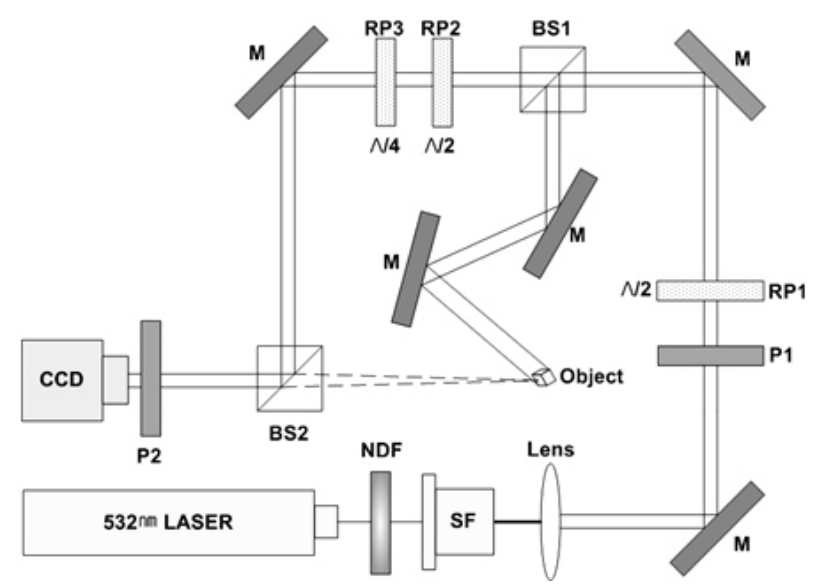

(a)

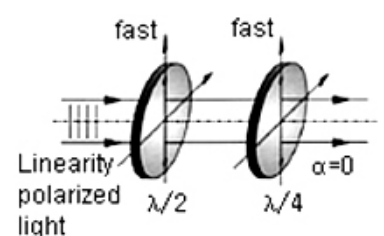

light
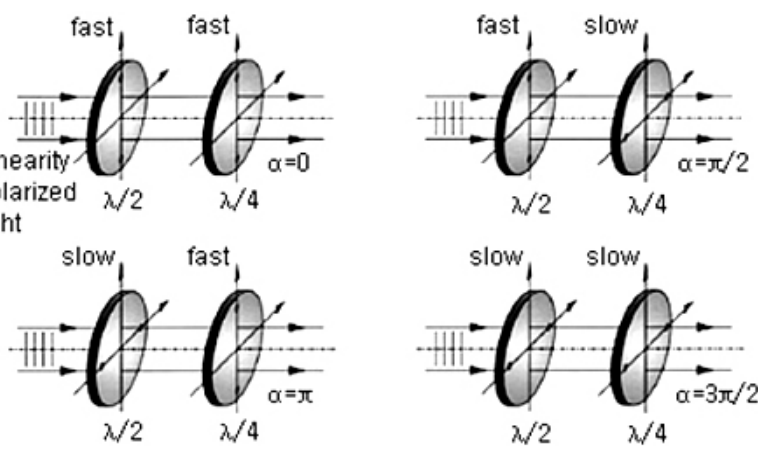

(b)

FIG. 4. (a) Arrangement for recording 4-step phase shifting digital hologram: SF, spatial filter; M, mirror; P1, P2, polarizer; NDF, neutral density filter; BS1, BS2, beam splitter; RP1, RP2, RP3, retardation plate.

(b) 4-step phase shifting using retardation plates.

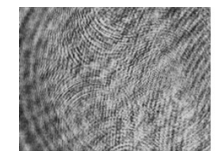

(a) $a=0$

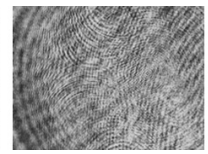

(b) $a=\pi / 2$

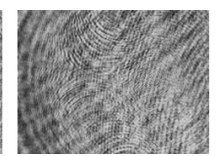

(c) $a=\pi$

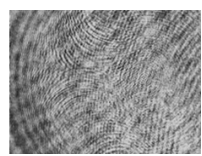

(d) $a=3 \pi / 2$
FIG. 5. Interference patterns of each phase with CCD camera.

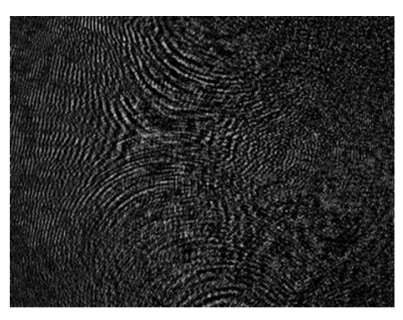

(a) $A_{E}$ (Amplitude)

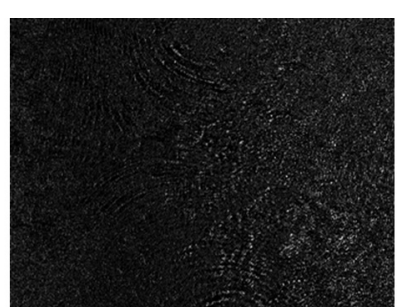

(b) $\phi_{E}$ (Phase)
FIG. 6. Calculated U(Object complex field). (a) $A_{E}$ (Amplitude) and (b) $\phi_{E}$ (Phase)

$$
\begin{aligned}
& \Delta x=\frac{\lambda z_{l}}{\delta_{x} N_{x}} \\
& \Delta y=\frac{\lambda z_{l}}{\delta_{y} N_{y}}
\end{aligned}
$$

where the sampling intervals at the CCD plane are $\delta_{x}$ and $\delta_{y}[20]$. Then the final sampling intervals of the elemental images at the $U_{d}$ plane are given by

$$
\begin{aligned}
& \Delta s=\frac{\lambda d}{\Delta x N_{x}}=\frac{d}{z_{l}} \delta_{x} \\
& \Delta t=\frac{\lambda d}{\Delta y N_{y}}=\frac{d}{z_{l}} \delta_{y}
\end{aligned}
$$

\section{EXPERIMENTAL RESULTS}

Figure 4(a) shows the experimental setup for recording a 4-step phase-shifting hologram based on Mach-Zehnder interferometer [21]. The Nd:YAG laser beam(532nm) passes through the NDF(Neutral Density Filter), the polarizer and the $\lambda / 2$ wave plate and then divided by a beam splitter into object and reference beams. The reference beam passes through the other $\lambda / 2$ wave plate and $\lambda / 4$ wave plate. The object beam illuminates the object after reflection by two mirrors. These two beams are put together by another beam splitter and their interference pattern is captured by the CCD camera. We used a coin located at $56.5 \mathrm{~cm}$ from the CCD as an object. The CCD camera used in the experiment has $3272 \times 2469$ pixels with $27 \mu \mathrm{m} \times 27 \mu \mathrm{m}$ pixel size. The phase-shifting of the reference beam is achieved by rotating the $\lambda / 2$ - and $\lambda / 4$ - wave plates, RP2 and RP3 in Fig. 4(a). By aligning different slow and fast axes with the direction of the polarization, 4-step phase values $a=0, \pi / 2, \pi$ and $3 \pi / 2$ can be produced with high accuracy as shown in Fig. 4(b) [17]. Figure 5 shows the interference patterns captured by the CCD for 4 phase shifts. From these 4 interference patterns, the complex object field is calculated by Eqs. (3) and (4) as shown in Fig. 6. The calculated values of the view angles of CCD camera plane are $\theta_{\mathrm{x}}=0.017^{\circ}$ and $\theta$ $\mathrm{y}=0.013^{\circ}$ respectively by Eq. (10).

Using the calculated complex object field, the elemental images for various parameters are synthesized. Figure 7 shows an elemental image generated assuming only one elemental lens. The parameters used in generating Fig. 7 are $f=4 \mathrm{~mm}, z_{l}=6 \mathrm{~cm},\left(x_{o}, y_{o}\right)=(2 \mathrm{~mm}, 0), W_{x}=W_{y}$ $=2 \mathrm{~mm}, \mathrm{~d}=4.032 \mathrm{~mm}$ where $W_{x}$ and $W_{y}$ are the lens size. We can see that a clear image of the object is reconstructed by the proposed method. The object image size measured in Fig. 7 is $597 \times 666$ pixels or $0.10834 \mathrm{~mm} \times$ $0.12087 \mathrm{~mm}$ considering the sampling interval calculated by Eq. (12). Note that because the actual size of the coin is about $1.5 \mathrm{~cm} \times 1.5 \mathrm{~cm}$ if we get the elemental 


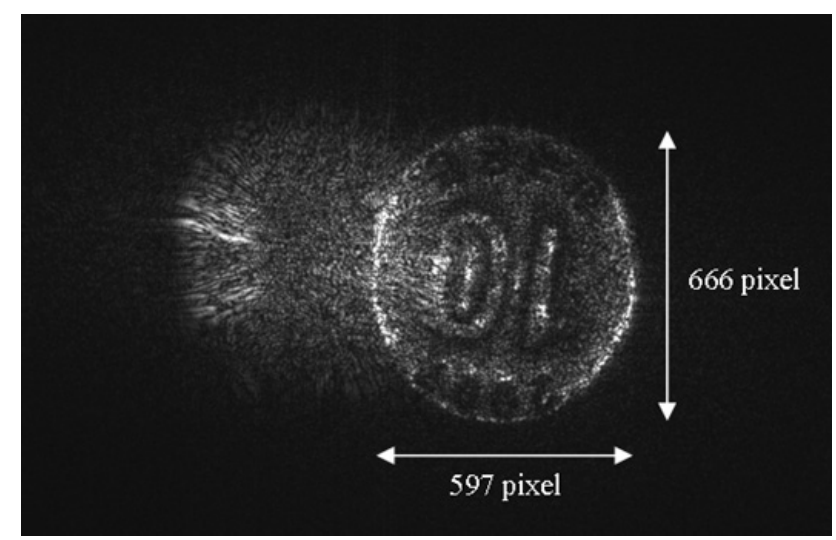

FIG. 7. The reconstructed elemental image in $\left(x_{o}, y_{0}\right)=$ $(0,0), W_{x}=W_{y}=2 \mathrm{~mm}, f=4 \mathrm{~mm}, z_{l}=6 \mathrm{~cm},: d=4.0329 \mathrm{~mm}$, full image size $=0.59381 \mathrm{~mm} \times 0.44808 \mathrm{~mm}$.

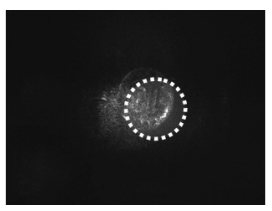

(a)

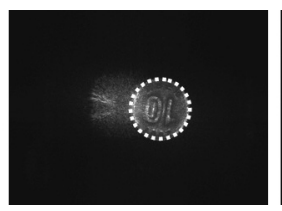

(b)

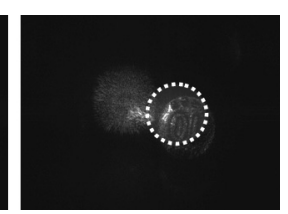

(c)
FIG. 8. The reconstructed elemental images in $z_{l}=7$ $\mathrm{cm}, f=5 \mathrm{~mm}, W_{x}=W_{y}=3 \mathrm{~mm} ; d=5.05263 \mathrm{~mm}$

(a) $\left(x_{o}, y_{0}\right)=(-3 \mathrm{~mm}, 3 \mathrm{~mm}),(\mathrm{b})\left(x_{o}, y_{o}\right)=(0,0)$ and (c) $\left(x_{o}\right.$, $\left.y_{o}\right)=(3 \mathrm{~mm},-3 \mathrm{~mm})$.

image optically with actual elemental lens of the same parameters, then its size will be [actual object size] $\times d /\left(z_{0}-z_{l}\right)=[0.015 \mathrm{~m}] \times 0.0040329 \mathrm{~m} /(0.565 \mathrm{~m}-0.06 \mathrm{~m})=$ $0.1198 \mathrm{~mm}$, which is in a good match with the measured value in Fig. 7. Therefore, we can confirm that the size of the elemental image produced by the proposed method is realistic for given parameters. The disparity between the elemental images is also verified experimentally. Figure 8 shows the generated elemental images for 3 different elemental lens locations $\left(x_{o}, y_{0}\right)$, i.e. $(0 \mathrm{~mm}, 0$ $\mathrm{mm}),(-3 \mathrm{~mm}, 3 \mathrm{~mm})$, and $(3 \mathrm{~mm},-3 \mathrm{~mm})$. The dotted line circle shown in Fig. 8 is the position of the elemental image when the elemental lens is at $(0 \mathrm{~mm}, 0 \mathrm{~mm})$. Since the separation between the elemental lenses is $3 \sqrt{2}$ $\mathrm{mm}$, the disparity should be [lens' separation] $\times \mathrm{d} /(\mathrm{zo}-\mathrm{zl})=$ $[3 \mathrm{~mm}] \times 5.05263 \mathrm{~mm} / 495 \mathrm{~mm}=0.0433 \mathrm{~mm}$. The disparity measured experimentally from Figs. 8(a) and (b) is (160 pixel, 148 pixel), or $\sqrt{\left(160^{2}+148^{2}\right.} \times 10^{4} \mathrm{~mm}=0.0425 \mathrm{~mm}$, considering the sampling interval. This measured disparity value $0.0423 \mathrm{~mm}$ is well matched to the expected value $0.0433 \mathrm{~mm}$, confirming the proposed method produces the elemental images of correct disparity. Figures 9-10 shows the generated elemental images for various elemental lens focal lengths $\mathrm{f}$, and longitudinal locations zl. The sizes of all images in Fig. 9 and Fig. 10 are normalized, considering their sampling interval, for easier comparison. It is observed from Figs. 9-10 that the

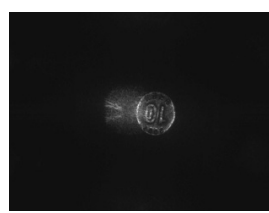

(a)

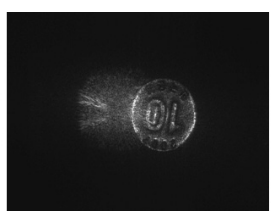

(b)

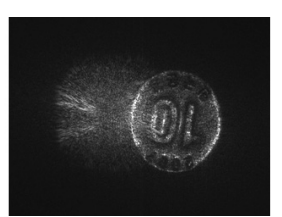

(c)
FIG. 9. The reconstructed elemental images in $z_{l}=5$ $\mathrm{cm},\left(x_{o}, y_{o}\right)=(0,0), W_{x}=W_{y}=3 \mathrm{~mm}$, image size $=0.53326$ $\mathrm{mm} \times 0.40239 \mathrm{~mm}$; (a) $f=3 \mathrm{~mm}, d=3.0181 \mathrm{~mm}$, (b) $f=5$ $\mathrm{mm}, d=5.0505 \mathrm{~mm}$ and (c) $f=7 \mathrm{~mm}, d=7.0993 \mathrm{~mm}$.

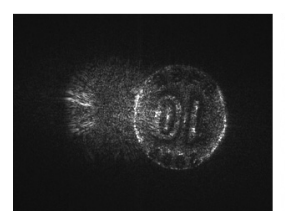

(a)

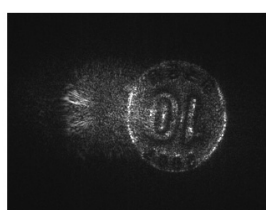

(b)

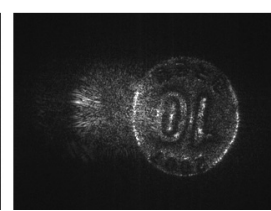

(c)
FIG. 10. The reconstructed elemental images in $f=4 \mathrm{~mm}$, $\left(x_{o}, y_{o}\right)=(0,0), W_{x}=W_{y}=2 \mathrm{~mm}$, image size $=0.29725 \mathrm{~mm} \times$ $0.2243 \mathrm{~mm}$; (a) $z_{l}=6 \mathrm{~cm}, d=4.0329 \mathrm{~mm}$, (b) $z_{l}=9 \mathrm{~cm}$, $d=4.035 \mathrm{~mm}$ and (c) $z_{l}=12 \mathrm{~cm}, d=4.0376 \mathrm{~mm}$

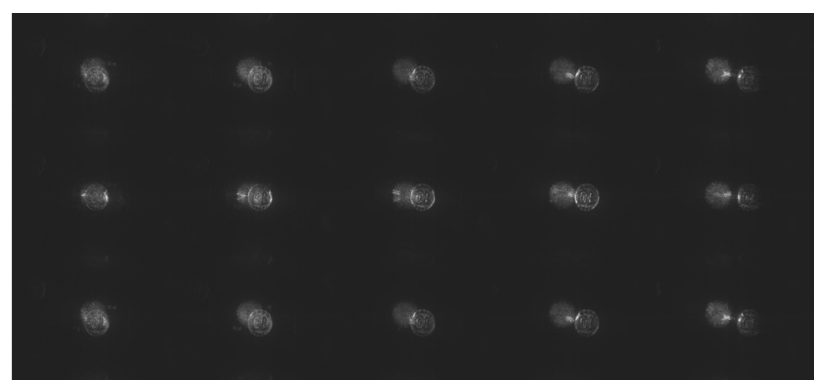

FIG. 11. The reconstructed elemental images in $z_{l}=5$ $\mathrm{cm}, f=4 \mathrm{~mm}, W_{x}=W_{y}=2 \mathrm{~mm}, d=4.0322 \mathrm{~mm}$

elemental images are generated correctly with given parameters by the proposed method. Finally, Fig. 11 shows the generated full set of elemental images assuming a lens array of the parameters $f=4 \mathrm{~mm}, z_{l}=5 \mathrm{~cm}$, $W_{x}=W_{y}=2 \mathrm{~mm}$. We can see the elemental images are generated clearly with the proper disparity and realistic sizes, confirming the feasibility of the proposed method. Although only $5 \times 3$ elemental images are shown in Fig. 11 , it is straightforward to increase the number of the elemental images. The circular noise seen in each elemental image might be produced by the unwanted reflection from the optical components used in the experimental setup. It is believed that this noise will be removed by a more refined experiment.

\section{DISCUSSION AND CONCLUSION}

In this paper, we proposed a method generating elemental images for integral imaging using the 4-step 
phase shifting digital hologram. After the complex field of the object is obtained by the 4-step phase shifting digital hologram, we propagate the complex object field numerically assuming a virtual lens array in the reconstruction space, resulting in the elemental images. We can control the parameters about elemental lens' focal length $\mathrm{f}$, propagated distance of obtained hologram $z$, elemental lens' transverse location $\left(x_{o}, y_{0}\right)$ and elemental lens' horizontal and vertical length $W_{x}$ and $W_{y}$. Since the hologram contains full 3D information of the object, we can generate the elemental images of the object for arbitrary lens array. Therefore from single acquisition of the object hologram, elemental images for any integral imaging display system can be synthesized regardless of its specifications. The result verifies the principle of the proposed method.

\section{ACKNOWLEDGMENT}

"This work was supported by the grant of the Korean Ministry of Education, Science and Technology" (The Regional Core Research Program / Chungbuk BIT Research-Oriented University Consortium)

\section{REFERENCES}

[1] Yamaguchi and T. Zhang, "Phase-shifting digital holography," Opt. Lett., vol. 22, no.16, pp. 1268-1270, 1997.

[2] Yamaguchi, T. Matsumura, and J. Kato, "Phase-shifting color digital holography,” Opt. Lett., vol. 27, no. 13, pp. 1108-1110, 2002.

[3] U. Schnars and W. Jüptner, "Direct recording of holograms by a CCD target and numerical reconstruction," Appl. Opt., vol. 33, no.2, pp. 179-181, 1994.

[4] J. W. Kang and C. K. Hong, "Three Dimensional Shape Measurement of a Micro Fresnel Lens with In-line Phaseshifting Digital Holographic Microscopy," J. Opt. Soc. Korea, vol. 10, no. 4, pp. 178-183, 2006.

[5] H. J. Lee and S. K. Gil, "Error Analysis for Optical Security by means of 4-StepPhase-Shifting Digital Holography," J. Opt. Soc. Korea, vol. 10, no. 3, pp. 118-123, 2006.

[6] F. Okano, H. Hoshino, J. Arai, and I. Yuyama, "Real-time pickup method for a three-dimensional image based on integral photography," Appl. Opt., vol. 36, no.7, pp. 1598-1603, 1997.

[7] S. Manolache, A. Aggoun, M. McCormick, N. Davies, and S. Y. Kung, "Analytical model of a three-dimensional integral image recording system that uses circular and hexagonal-based spherical surface microlenses," J. Opt. Soc. Am. A, vol. 18, no. 8, pp. 1814-1821, 2001.

[8] J.-H. Park, Y. Kim, J. Kim, S.-W. Min, and B. Lee, "Three-dimensional display scheme based on integral imaging with three-dimensional information processing," Opt. Exp., vol. 12, no.24, pp. 6020-6032, 2004.

[9] S.-H. Shin and B. Javidi, "Speckle reduced threedimensional volume holographic display using integral imaging," Appl. Opt., vol. 41, no.14, pp. 2644-2649, 2002.

[10] Y. Kim, J.-H. Park, H. Choi, S. Jung, S.-W. Min, and B. Lee, "Viewing-angle-enhanced integral imaging system using a curved lens array," Opt. Exp., vol. 12, no. 3, pp. 421-429, 2004.

[11] M. Martınez-Corral, B. Javidi, R. Martıjnez-Cuenca, and G. Saavedra, "Integral Imaging with Improved Depth of Field by Use of Amplitude-Modulated Microlens Arrays," Appl. Opt., vol. 43 no. 31, pp. 5806-5813, 2004.

[12] Y. Kim, J.-H. Park, H. Choi, J. Kim, S.-W. Cho, and B. Lee "Depth-enhanced three-dimensional integral imaging by use of multilayered display devices," Appl. Opt., vol. 45, no. 18, pp. 4334-4343, 2006.

[13] J.-S. Jang and B. Javidi, "Improvement of Viewing Angle in Integral Imaging by Use of Moving Lenslet Arrays with Low Fill Factor,” Appl. Opt., vol. 42, no. 11, pp. 1996-2002, 2003.

[14] D.-H. Shin and E.-S. Kim, "Computational Integral Imaging Reconstruction of 3D Object Using a Depth Conversion Technique," J. Opt. Soc. Korea, vol. 12, no. 3, pp. 131-135, 2008.

[15] B. Javidi and S.-H. Hong, "Three-Dimensional Holographic Image Sensing and Integral Imaging Display," Journal of Display Technology, vol. 1, no. 2, pp. 341-346, 2005.

[16] S.-C. Kim, P. Sukhbat, and E.-S. Kim, "Generation of three-dimensional integral images from a holographic pattern of 3-D objects," Appl. Opt., vol. 47, no. 21, pp. 3901-3908, 2008.

[17] T. Tajahuerce, O. Matoba, S. Verral, and B. Javidi, "Optoelectronic information encryption with phase-shifting interferometry”, Appl. Opt., vol. 39, no.14, pp. 23132320, 2000.

[18] T.-C. Poon, Digital Holography and Three-Dimensional Display: Principles and Applications (Springer Science+ Business Media, Inc., New York, USA, 2006) Chapter 5.

[19] J. W. Goodman, Introduction to Fourier Optics, 2nd ed. (McGraw-Hill, New York, USA, 1996) Chapter 5.

[20] T. Nakatsuji and K. Matsushima, "Free-viewpoint images captured using phase-shifting synthetic aperture digital holography," Appl. Opt., vol. 47, D136-D143, 2008.

[21] C. P. McElhinney, B. M. Hennelly, and T. J. Naughton, "Extended focused imaging for digital holograms of macroscopic three-dimensional objects," Appl. Opt., vol. 47, D71-D79, 2008. 